\title{
Arbor
}

\section{El Instituto Hidrográfico de la Marina}

\section{Francisco J. Pérez Carrillo de Albornoz}

Arbor CLXXIII, 682 (Octubre 2002), 365-383 pp.

Se expone en este artículo la amplia labor que desarrolla el Instituto Hidrográfico de la Marina(IHM) y su participación en múltiples programas y proyectos nacionales e internacionales, representando a España y manteniendo relaciones permanentes de colaboración con diversas Organizaciones, Comisiones y Comités de carácter supranacional.

Tras una sintesis histórica del IHM, cuyos orígenes en el Padrón Real de 1508 le otorgan la primacía mundial como Instituto Hidrográfico, se describen detalladamente las actividades que desarrolla el Instituto en las diferentes áreas de su competencia (Hidrografía, Geodesia y Fotogrametría, Cartografía, Navegación, Oceanografía), destacando el intenso y continuado trabajo que realizan sus buques hidrográficos y el proceso de transformación que se está llevando a cabo para implantar las modernas tecnologías en todos estos campos de actuación.

Haciendo honor a la cita de que «Europa aprendió a navegar en libros españoles», se recogen también en este artículo las numerosas publicaciones que continúa editando el Instituto, como responsable que es de mantener actualizada la cartografía náutica y sus correspondientes avisos y ayudas a la navegación, para finalizar con una referencia a la labor docente que desarrolla su Escuela de Hidrografía.

\section{Antecedentes históricos}

Para encontrar los orígenes de la actividad hidrográfica y de la cartografía española hay que remontarse a los siglos XIII, XIV y XV, 
y está íntimamente relacionada con las singladuras de las marinas de Aragón y Castilla y el incremento de la actividad comercial en el mar Mediterráneo. Es en esta época cuando nace la escuela catalana, en su mayor parte formada por mallorquines, y que ostenta la primacía mundial del progreso cartográfico.

Hacia finales del siglo XIV aparecen dos figuras fundamentales de esta escuela: Abraham y Jafuda Cresques, siendo Abraham el autor del Gran Atlas Catalán, publicado en 1.375, que comprende desde las costas españolas y africanas hasta el litoral de China.

La actividad hidrográfica y cartográfica se intensifica con el descubrimiento del Nuevo Mundo por la necesidad de reflejar en las cartas los nuevos descubrimientos y por el cambio de la forma de navegar, que pasa de una navegación de cabotaje y costera a otra de altura y oceánica.

En el año 1.500 el cartógrafo Juan de la Cosa publica la primera carta de navegación del Nuevo Mundo, lo que supone el nacimiento de la escuela sevillana. Esta carta, que se conserva en el Museo Naval de Madrid, marca un hito muy importante en el desarrollo de la actividad cartográfica española, de la que pueden citarse como acontecimientos más destacados a partir de esa fecha los siguientes:

En 1.503 se funda la Casa de Contratación de Sevilla, que constituye el centro de todos los estudios geográficos y náuticos, y se crea el cargo de Piloto Mayor, desempeñado por hombres tan ilustres como Juan de la Cosa y Américo Vespucio.

En 1.508, Fernando El Católico ordena la creación del Padrón Real, especie de Carta Patrón para uso de todos los pilotos, donde debían registrarse todos los nuevos descubrimientos y rutas marítimas. Este Padrón Real, por su organización, función y actividades, puede considerarse como el más antiguo Instituto Hidrográfico del mundo.

En 1681 se funda en Sevilla el Colegio de San Telmo, para la formación de los marinos de oficio grabadores y la confección de cartas náuticas y derroteros.

Desde 1.725, la hidografía fue uno de los temas más estudiados en la Academia de Guardiamarinas de Cádiz, fundada por Felipe V, pasando a ser la construcción de cartas náuticas responsabilidad exclusiva de la Armada.

De 1.735 a 1.744 Jorge Juan y Antonio de Ulloa participan en la expedición científica de la medición del Arco de Meridiano en Perú.

De 1.783 a 1.788 el ilustre Brigadier Vicente Tofiño lleva a cabo el levantamiento de los puertos y costas de la Península, dando como fruto el Atlas Marítimo de España, con sus correspondientes derroteros. Esta obra es el fundamento de la cartografía náutica española moderna 
$\mathrm{y}$ muchas de sus cartas permanecieron en vigor hasta bien entrado el siglo XX.

Asimismo, entre 1.789 y 1.794 tiene lugar la expedición Malaspina, que fue la más ambiciosa y completa hasta entonces realizada y que tuvo, entre otros, el objetivo de mejorar la cartografía de todas las posesiones españolas, elaborando un total de 185 cartas y 200 borradores.

Con objeto de atender a la conservación de estos trabajos originales y a su reproducción para darlos a conocer a los navegantes, se impone como ineludible la creación de un centro científico, naciendo así la Dirección de Hidrografía en 1.797, en Madrid.

La ingente labor realizada por este centro en algo más de un siglo de existencia se debe fundamentalmente al impulso de una serie de directores tan prestigiosos como Espinosa, Bauzá, Navarrete, Conde de Aubarede, y Lasso de la Vega. Concretamente, este último inició en 1.851 la publicación de las hojas de Avisos a los Navegantes tal y como hoy la conocemos, y en las que empezaron a darse noticias sobre las alteraciones en las luces de faros.

En 1.927 se suprime la Dirección de Hidrografía y se crea en el Observatorio de Marina la $4^{a}$ Sección, más conocida como Servicio Hidrográfico de la Armada, asumiendo el Director del Observatorio el cargo de Director de Hidrografía.

En 1908 se sanciona y reconoce de forma oficial la Especialidad de Hidrografía en la Armada, estableciéndose en el vapor Urania la academia para su enseñanza.

En 1921, España fue uno de los dieciocho estados fundadores del «Bureau Hidrográfico Internacional», organismo creado para fomentar la cooperación y normalización entre los Servicios Hidrográficos.

En 1.943, y ante la necesidad de potenciar la hidrografía y recuperar su unidad, nace el Instituto Hidrográfico.

\section{Cometidos}

Por Decreto 3.853/1.970 de 31 de Diciembre, se otorga al Instituto Hidrográfico de la Marina (IHM) la misión de velar por la seguridad de la navegación, en sus aspectos de obtener y difundir información sobre la mar y el litoral, y contribuir al progreso de la ciencia náutica.

En cumplimiento de su misión, tiene como cometidos principales los siguientes:

- Efectuar los levantamientos hidrográficos/oceanográficos necesarios para el estudio del relieve submarino en nuestras costas 
y zonas marítimas, o en otras que el Estado español considere oportuno como consecuencia de convenios internacionales.

- Elaboración de cartas náuticas y redacción de libros y documentos de ayudas a la navegación, así como su edición y distribución.

- Elaboración de aquella cartografía temática y ejecución de programas de investigación que, por necesidades de apoyo a la Fuerza, se le asignen con un destino específico para la Armada.

Asimismo, por Ley 7/1.986 de Ordenación de la Cartografía, se determina que será competencia del IHM la formación y conservación de la Cartografía Náutica básica, con lo que se confiere a su producción la categoría de cartografía de Estado y documentación oficial de preceptiva utilización para el navegante.

Estos cometidos abarcan tres áreas fundamentales:

- Adquisición de datos: Incluye los levantamientos hidrográficos y actividades oceanográficas sistemáticas en zonas de responsabilidad nacional e internacional, así como la consulta de toda otra fuente de información cartográfica y náutica.

- Tratamiento de Datos y Producción: Incluye la edición de cartas y publicaciones náuticas y la elaboración de informes y documentos descriptivos oceanográficos, así como el mantenimiento de bases de datos georeferenciados.

- Investigación y Desarrollo: Que contempla tanto la investigación aplicada en las áreas de Geodesia, Geofísica y Oceanografía, como el desarrollo de las infraestructuras de adquisición de datos y sistemas de producción:

La actividad en estas tres áreas ha de orientarse armónicamente, de manera que puedan determinarse y divulgar las siguientes características de cada zona geográfica concreta:

- Configuración de la costa.

- Relieve submarino y naturaleza del fondo, con especial énfasis en aquellos accidentes que constituyen un peligro para la navegación.

- Composición del subsuelo y sedimentos.

- Comportamiento físico / químico de las masas de agua.

- Existencia y estado de las Ayudas a la Navegación.

Para llevar a cabo estas funciones, el IHM se encuentra dividido orgánicamente en cinco Secciones Técnicas:

- Hidrografía.

- Geodesia y Fotogrametría.

- Cartografía. 
- Navegación.

- Oceanografía.

Asimismo existe un Centro de Cálculo, que presta apoyo informático a todo el Instituto y a la Escuela de Hidrografía, esta última también ubicada dentro del IHM.

Se describen a continuación cada una de estas Secciones Técnicas, haciendo especial hincapié en cuál es su cometido, cómo lo realizan y cuál es su futuro.

\section{Sección de Hidrografía}

La Sección de Hidrografía, es la encargada de proyectar, dirigir y comprobar los levantamientos hidrográficos necesarios para garantizar la seguridad de la navegación en nuestras zonas de responsabilidad.

España posee una línea de costa con una longitud total de 5.300 $\mathrm{Km}$ y una extensión virtual de su ZEE de 3,4 millones de $\mathrm{Km}^{2}$.

Las necesidades de adecuación de toda área a hidrografiar exige esfuerzos y recursos completamente diferentes en función del tráfico y márgenes de seguridad admisibles en cada zona, de acuerdo con lo especificado en la publicación especial S-44 de la Organización Hidrográfica Internacional (OHI), en vigor desde 1.997.

Se pueden agrupar estas zonas de la manera siguiente:

- Puertos, zonas de fondeo, canales de acceso a puertos y canales críticas, donde debe garantizarse un recubrimiento total del fondo con sistema multihaz y sonar de barrido lateral. La extensión total de esta zona se puede considerar de $20.000 \mathrm{Km}^{2}$ Estos levantamientos, de los que se encontraban adecuadamente efectuados el $96 \%$, de acuerdo con la nueva doctrina de la S-44 hay que efectuarlos de nuevo.

- Áreas costeras hasta el veril de los 100 metros, no incluidas en el punto anterior, donde debe quedar asegurada la navegación marítima. La extensión total de estas áreas es de $74.000 \mathrm{Km}^{2}$, de las que se pueden considerar adecuadamente levantadas un $95 \%$.

- Áreas continentales, con profundidades comprendidas entre 100 y 200 metros. La extensión total es de $50.000 \mathrm{~km}^{2}$, de los que se pueden considerar adecuadamente levantados un $96 \%$.

- Otras áreas hasta las 200 millas naúticas donde las profundidades superan los 200 metros. Hacen un total de 3,244 millones de $\mathrm{Km}^{2}$ si se considera lo que podría ser reclamado por España como ZEE. De ellos se llevan levantados un $7 \%$. 
Para realizar estas misiones, el IHM cuenta con los seis buques hidrográficos Malaspina, Tofiño, Cástor, Póllux, Antares, y Rigel, que efectúan, cada uno de ellos, una media de 165 días de mar al año. Adicionalmente se dispone del BIO Hespérides durante un mes al año, para realizar las campañas contempladas en el Plan de Investigación Hidrográfica y Oceanográfica de la Zona Económica Exclusiva (ZEE), fundamentalmente para batimetría multihaz en aguas profundas.

El pasado 30 de noviembre, se le entregó al Instituto Hidrográfico una lancha hidrográfica con todos los equipos del Sistema Hidrográfico Integrado instalados y que constituye la primera unidad de un grupo de tres, que relevarán a los ya cansados Cástor y Póllux, entregados a la Armada en 1.965 .

Una vez adquiridos los datos a bordo de los buques, la Sección de Hidrografía los procesa para elaborar los correspondientes parcelarios, base de la cartografía náutica. Los datos digitales así obtenidos son incorporados a la Base de Datos Hidrográficos, en la que también se integran los de los antiguos parcelarios.

Asimismo, esta Sección es responsable de la compilación de toda la información cartográfica y ambiental precisa para la conducción de las operaciones de la Guerra de Minas, en una publicación que se distribuye entre las Armadas aliadas y elaborada de acuerdo con la normativa de la OTAN.

En diciembre de 1.999 se instaló en el B/H Rigel un sondador multihaz de aguas someras (hasta 200 metros) de alta tecnología, que permite al IHM cumplir con los requisitos fijados por la Organización Hidrográfica Internacional para los levantamientos de puertos, canales de acceso a puertos y canales críticas para la navegación.

Con la incorporación de este sistema multihaz ha empezado una auténtica revolución en la hidrografía, ya que con el recubrimiento total deja de ser una ciencia estadística y desaparece el concepto de escala a efectos de planeamientos de trabajos, utilizando una Base de Datos que almacena gran cantidad de información en cada levantamiento (se pueden obtener hasta 4.500 datos de sondas con sus correspondientes metadatos cada segundo).

\section{Sección de Geodesia y Fotogrametría}

La Sección de Geodesia y Fotogrametría tiene como misión fundamental la de proporcionar toda la información terrestre necesaria para la producción de la cartografía naútica y apoyo a los trabajos de los buques hidrográficos. 
- La producción cartográfica requiere de esta Sección toda la información planimétrica y altimétrica del terreno, detalle de la línea de costa y parámetros de transformación entre sistemas de referencia.

- El apoyo a los buques se realiza con el aporte de toda la información terrestre necesaria para el preciso desarrollo de los trabajos hidrográficos, es decir, la información relativa a la red geodésica nacional mantenida por el Instituto Geográfico Nacional y a la red de control hidrográfico establecida y mantenida por esta Sección, remitiendo a los buques los parámetros de transformación entre sistemas geodésicos.

Todos estos campos de trabajo no dejan de lado el estudio, investigación y colaboración con otros organismos, nacionales y extranjeros, para contribuir con ellos al progreso de las ciencias geodésicas.

Además de estas misiones principales, y que son su razón de ser, esta Sección presta apoyo a otras unidades de la Armada, tales como las de Medidas Contra Minas, proporcionándoles la información geodésica necesaria para el posicionamiento preciso durante el desarrollo de sus operaciones específicas. También se colabora con cualquier otra unidad o dependencia que necesite del conocimiento exacto de posiciones y medidas sobre el terreno.

Para llevar a cabo su labor, esta Sección cuenta con dos Subsecciones estrechamente relacionadas entre sí:

- La Subsección de Fotogrametría, que se encarga de elaborar proyectos y establecer directrices para la obtención de los levantamientos fotogramétricos que proporcionan información planimétrica del terreno, así como la coordinación con otros centros cartográficos de las Fuerzas Armadas, nacionales y autonómicos, para intercambio de productos geográficos y evitar duplicidades en la generación de información.

- La Subsección de Geodesia, que realiza el estudio de las superficies geodésicas de referencia, de las redes geodésicas que sobre ellas se establecen, y genera los parámetros de transformación entre unas y otras.

Independientemente de todos los trabajos que se realizan en la Sección para cumplir de forma cotidiana con la misión encomendada, merecen ser destacados, por su relevancia, los siguientes:

\section{Obtención de parámetros de transformación ED50 a WGS84*}

Conforme avanza la Geodesia, se van elaborando sistemas de referencia cada vez más precisos, lo que conlleva la sustitución 
de unos por otros. Pero esta sustitución no es inmediata, sino que durante un período más o menos largo conviven los dos sistemas, hasta que la nueva referencia se adopta a todos los niveles y la antigua deja de ser necesaria.

Esto es lo que sucede en la actualidad dentro de nuestro marco geográfico. Nos encontramos en un período de transición entre el sistema regional ED50 y el global y más preciso WGS84. Lo mismo ocurre en todas las regiones del planeta. Por tanto, muchos proyectos cartográficos se encuentran normalmente con la necesidad de trabajar con elementos referidos a los dos sistemas y obtener un producto final en uno sólo de ellos. La tendencia actual, en el IHM, es elaborar estos productos en el sistema WGS84. De esta forma, las posiciones obtenidas por el navegante con el sistema GPS pueden plasmarse directamente sobre la carta.

En este sentido, el IHM, está finalizando una serie de campañas geodésicas para la obtención de parámetros de transformación entre estos sistemas para las distintas zonas del litoral nacional, mediante la observación geodésica de vértices con sistema GPS geodésico, con la idea de disponer conjuntos de dobles coordenadas WGS84 y ED50 para cada uno de ellos. De esta forma, con los algoritmos matemáticos adecuados, se obtienen los parámetros correspondientes.

Gracias a estos trabajos, el IHM es el primer organismo que, a nivel oficial y nacional, ha publicado su cartografía para la Península en el sistema de referencia WGS84.

\section{Implantación de un sistema de fotogrametría digital.}

La actualización cartográfica necesita una herramienta de trabajo muy precisa para las tareas de control de calidad de la información cartográfica gráfica, procedente de la restitución numérica. La obtención del detalle de la línea de costa del litoral español se realiza exclusivamente en este centro, y para que esta información sea lo más fiable posible, hay que utilizar los medios más modernos en lo que a restitución fotogramétrica se refiere.

Durante los últimos años, y gracias al avance de la informática, la fotogrametría ha experimentado importantes cambios con la 


\section{El Instituto Hidrográfico de la Marina}

utilización de imágenes digitales; tales efectos han dado lugar a una nueva generación de instrumentos que son las Estaciones de Fotogrametría Digital, cuyo empleo se ha extendido a todas las tareas relacionadas con esta actividad.

Después de 25 años de trabajo con los restituidores analógicos, y sin haber pasado por la generación de restituidores analíticos que empiezan a quedarse anticuados, las necesidades actuales de producción requieren un sistema actual, versátil, eficaz e integrado dentro de un sistema cada vez más compacto en cuanto a homogeneidad interna de trabajo, y abierto a su vez a fuentes externas de información.

La integración reciente de una Estación Digital dentro del sistema de producción cartográfica del IHM, ha conseguido una gran fiabilidad y agilización en los levantamientos fotogramétricos e intercambio de información digital, que repercute en la seguridad, calidad y ritmo de producción.

\section{Sección de Cartografía}

Una de las principales actividades del Instituto Hidrográfico de la Marina es la producción y mantenimiento de la cartografía que constituye su Catálogo de Cartas Náuticas, tanto en formato tradicional (papel) como en formato ENC (carta electrónica).

\section{La Carta Náutica}

El objetivo principal de una carta náutica es auxiliar al navegante en un tránsito seguro.

La «carta» contiene por tanto todo lo que se conoce del fondo marino; muestra la profundidad por medio de sondas y veriles, y destaca los bajos y otras posibles obstrucciones, tales como pecios o cables submarinos.

Asimismo contiene la configuración de la línea de costa y la franja de tierra adyacente con aquellas características topográficas y puntos conspicuos que son notables desde la mar. Figuran todas las ayudas a la navegación existentes en la zona (faros, boyas, balizas transpondedoras, etc.), junto con una abreviatura de sus características. Incluye los Dispositivos de Separación de Tráfico, así como derrotas recomendadas y fondeaderos designados o aconsejados. Y refleja también aquellas zonas restringidas o prohibidas, tanto para algún tipo de navegación como para alguna clase de barco o tipo de pesca. 
Es decir, una carta náutica incluye toda aquella información que se considera que el navegante puede necesitar en un momento determinado. Aquella información que no pueda contener la carta figura en las publicaciones náuticas complementarias, como son los Derroteros y Libros de Faros.

Las cartas náuticas contienen mayor o menor detalle de información dependiendo del propósito para el que van a ser utilizadas. Por ello, y de acuerdo con su finalidad -que va ligada a un margen de escalas-, existen los siguientes tipos:

- Generales, realizadas a escala menor de 1:3.000.000, dedicadas al planeamiento de navegaciones oceánicas.

- Arrumbamiento, de escalas comprendidas entre 1:200.000 y 1:3.000.000, para su empleo en navegaciones a distancias medias.

- Costeras, realizadas a escalas comprendidas entre 1:50.000 y 1:200.000, diseñadas para la navegación de cabotaje.

- Aproches, editadas a escalas entre 1:25.000 y 1:50.000, proyectadas para su empleo en las entradas en puertos o pasos angostos.

- Puertos o Portulanos, realizadas a escalas mayores que 1:25.000, donde se muestran con detalle las características e instalaciones existentes en un determinado puerto.

\section{La producción cartográfica}

En el pasado más reciente los desarrollos efectuados en la producción cartográfica del Instituto fueron dirigidos hacia la obtención directa y completa de los cuatro originales: negro, azul, amarillo y violeta en colores sólidos que pudiesen ir directamente a su pasado a planchas off-set para el proceso de estampación, evitando así las labores de esgrafiado, separación de colores a mano, y pegado de textos y símbolos (stripping) que se venían realizando.

Conseguido esto, los esfuerzos se dirigieron a intentar cerrar la cadena digital en la producción de una carta náutica. Es decir, el objetivo era conseguir que la compilación de toda la información que da lugar a la carta náutica en un documento que denominamos minuta, se realizase en un entorno digital. Para ello se emplearon estaciones de trabajo que, utilizando programas típicos de diseño asistido (CAD), adecuados a la producción de cartografía náutica, obtienen una minuta digital a partir de ficheros digitales provenientes, fundamentalmente, de las secciones de Fotogrametría e Hidrografía. Todos estos procesos van unidos a la implantación de una red interna digital que enlaza 
a las diversas secciones de este Instituto y al desarrollo de un Sistema de Información Geográfica para la producción y mantenimiento de la colección de cartas náuticas.

Actualmente todas las nuevas cartas se realizan por medios automatizados.

\section{La Carta Náutica electrónica}

A partir de los progresos obtenidos por medio de la utilización de ficheros digitales y ordenadores en la producción de cartografía náutica en la forma tradicional, es decir en papel, había que dar el siguiente paso de obtener un producto digital para su uso directo por el navegante.

El tráfico marítimo es, por su propia naturaleza, internacional. Por eso la cartografía náutica está sometida a estándares y reglas de uniformidad de carácter universal. Así como la carta de papel se fue normalizando a lo largo del tiempo, debido fundamentalmente a los trabajos efectuados en el seno de la Organización Hidrográfica Internacional $(\mathrm{OHI})$, la carta náutica electrónica ya nace normalizada. Todos los países miembros de esta organización decidieron a priori cuál iba a ser el estándar para producir este tipo de cartografía.

Estas cartas náuticas electrónicas están diseñadas para ser utilizadas en unos equipos denominados ECDIS (Electronic Chart Display and Information System), o sistemas que montados en los puentes de los barcos presentan al navegante en un formato normalizado la carta náutica electrónica. Además, permiten poder extraer información de los elementos cartográficos que la forman, habilitando en su caso la generación de alarmas si se aproxima algún peligro o restricción en su navegación. Su funcionamiento, es decir, los requerimientos que debe tener tanto físicos como del programa de navegación, debe asimismo cumplir una normativa, regulada en este caso por la Organización Marítima Internacional.

Las cartas náuticas electrónicas producidas por el IHM se envían para su revalidación a un Centro Regional Europeo de Coordinación y Control (RENC), donde se unen con las elaboradas por otros Servicios Hidrográficos Oficiales para formar una base de datos que es puesta a disposición del navegante a través de una red de distribuidores.

\section{Actualizaciones}

Una parte fundamental, si no la más importante, de la calidad de una carta náutica, es el nivel a que se mantiene la actualización 
de aquella información que contiene y que está relacionada con la seguridad de la navegación.

Para ello, a partir de toda la información recibida en la Sección de Cartografía, se elaboran las correcciones pertinentes a las cartas náuticas editadas, y éstas se reúnen en el grupo semanal de Avisos a los Navegantes para su distribución. El navegante de esta forma puede tener su carta actualizada.

En el caso de las cartas náuticas electrónicas, estas correcciones toman la forma de ficheros digitales que se envían al citado Centro Regional de Coordinación para Europa, para su distribución. El navegante puede obtenerlas a través de comunicaciones vía satélite, facilitando de esta forma el mantenimiento actualizado de su colección de cartas.

\section{Sección de Navegación}

Se podría definir como una Sección de producción dentro del Instituto Hidrográfico, al igual que la Sección de Cartografía. Sus misiones fundamentales son:

- La edición y mantenimiento de todas aquellas publicaciones que complementan a las cartas náuticas, y la publicación de Avisos a los Navegantes, ya sea actuando como Coordinador Nacional de Avisos Costeros, o como Coordinador de Avisos de la NAVAREA III (Mediterráneo y Mar Negro), una de las 16 zonas en que se divide la Tierra, cuya responsabilidad corresponde al IHM a efectos de Avisos a los Navegantes.

- Igualmente se encarga de la publicación del grupo semanal de Avisos, que permite el mantenimiento de las cartas y publicaciones del Instituto.

Una descripción de las publicaciones más interesantes puede ser la siguiente:

\section{Libros de Faros}

Es un catálogo, publicado en dos volúmenes cada año y medio, en un formato internacionalmente aceptado, que contiene y describe todas las luces, señales fónicas y de balizamiento existentes en las siguientes zonas: desde el río Bidasoa en el Cantábrico hasta la frontera francesa en el Mediterráneo; desde la frontera Argelia/Túnez hasta Cabo Verde 
(Senegal), en África; y las costas y aguas de los archipiélagos de Baleares, Canarias, Azores, Madeira y Cabo Verde.

\section{Libro de Radioseñales}

Es una publicación semejante a la anterior, dedicada a todas las instalaciones de radioayudas a la navegación: radiofaros, radiogoniómetros, estaciones costeras, sistemas de posicionamiento electrónico, etc...También sigue un formato normalizado internacionalmente y su periodicidad es bienal.

\section{Derroteros}

Contienen una descripción literal de la costa, tal como es percibida por el navegante desde la mar, con énfasis en todo aquello que no puede ser gráficamente representado en una carta; en especial, zonas de peligros, condiciones meteorológicas y oceanográficas reinantes, instrucciones para tomar los puertos y fondeaderos, capacidad e instalaciones de los puertos, etc...

Se trata de 4 derroteros en 6 tomos que abarcan, desde la frontera del Bidasoa hasta Cabo Verde en el Atlántico, y desde la frontera francesa hasta la argelina-tunecina en el Mediterráneo.

\section{Avisos a los Navegantes}

Para permitir las actualizaciones de las cartas náuticas, así como para informar al navegante de cuantas incidencias puedan suponer un peligro para la navegación, existe todo un mecanismo de difusión de la información urgente: el Boletín semanal de Avisos a los Navegantes y el Sistema de Radioavisos a la Navegación:

\section{- Boletín de Avisos a los Navegantes}

Es un boletín impreso, de periodicidad semanal, que se remite por correo a los suscriptores. Permite al navegante mantener al día todas sus cartas y publicaciones náuticas de forma coherente y completa, sin exigir de él gran esfuerzo.

Es muy importante, para el usuario de las cartas y publicaciones náuticas, estar suscrito al Boletín de Avisos a los Navegantes, como forma de mantenerlas al día. Actualmente está en estudio la inclusión de todos los Avisos a los Navegantes en una página Web, de manera 
que el usuario, en todo momento, pueda extraer todos los avisos en vigor que afecten a su carta o publicación concreta.

- Radioavisos

En atención al ámbito geográfico al que afectan, pueden ser:

- Locales, en el dominio interno de un puerto, en general afectan únicamente al tráfico interior.

- Costeros, en el entorno del litoral y hasta unas 200 millas de la costa. Interesan a los buques en tránsito entre puertos $\mathrm{y}$ son coordinados a nivel nacional por el IHM.

- «Navárea», para zonas de alta mar y en rutas de tráfico internacional. Dado que en alta mar no puede hablarse de responsabilidades nacionales, la Organización Marítima Internacional (OMI) y la OHI han distribuido los océanos a estos efectos en un total de 16 áreas, conocidas como NAVAREAS. Cada una de ellas tiene un coordinador que centraliza y difunde los radioavisos que afectan a toda la zona. El IHM es el Coordinador de la Zona Navárea III, cuyo ámbito es el Mediterráneo y Mar Negro.

La misión del IHM, como Coordinador de Radioavisos Costeros y de Radioavisos Navárea, consiste en recibir información, evaluarla, redactar el radioaviso siguiendo formatos normalizados y encaminarlo a las emisoras correspondientes, controlando su emisión.

Para canalizar todo este flujo de información existe una red de datos (frame relay), red Mercurio propiedad de Telefonica, que enlaza todos los centros que de una u otra forma intervienen en la «Información de Seguridad Marítima» (Salvamento, Información Meteorológica y Radioavisos a los Navegantes): Centros de Control de Salvamento, IHM, Instituto Meteorológico Nacional, Estaciones Radiotransmisoras Costeras, etc.

Como medios de difusión de las distintas clases de Radioavisos se emplean los siguientes:

- Naváreas: Satélite de comunicaciones marítimas Inmarsat C, de impresión automática a bordo. Estos radioavisos se dan únicamente en lengua inglesa.

- Costeros: Estaciones Navtex próximas ( de impresión automática y sólo en lengua inglesa) y por fonía, en VHF y HF, a través de las estaciones radiocosteras de Telefónica, usando en este caso las lenguas inglesa y española.

- Locales: emisoras locales de VHF, y HF en inglés y español. 


\section{Sección de Oceanografía}

Desde el punto de vista de la Oceanografía, una de las principales misiones del Instituto Hidrográfico es el estudio de las mareas para su aplicación a la cartografía náutica y al Anuario de Mareas. Por ello, es necesario contar, por una parte, con largas series de datos de marea para mantener el Anuario de Mareas y, por otra, con una extensa red de puntos donde quede referido el Cero Hidrográfico, Datum vertical o Nivel de Reducción de Sondas. Estas dos necesidades han llevado a la Sección de Oceanografía a desarrollar dos líneas de actuación paralelas, una con mareógrafos permanentes y otra con mareógrafos portátiles.

El Instituto Hidrográfico participa en el programa RIMA (Integración de Redes Mareográficas) junto al Instituto Geográfico Nacional, al Instituto Español de Oceanografía y al Ente Público Puertos del Estado. Este programa, que nació como proyecto de investigación financiado por la CICYT, con la finalidad de armonizar las redes de mareógrafos permanentes gestionados por los distintos organismos, implantar el Cero Hidrográfico como nivel de referencia en todos los puntos de observación e identificar las carencias nacionales en lo relativo a la observación de las mareas, permanece bajo la forma de programa, sin financiación extraordinaria, proporcionando un marco adecuado para el flujo de la información. El Instituto Hidrográfico ha cubierto áreas con déficit en la observación de las mareas instalando mareógrafos permanentes en los puertos de Pasajes y Burela. En un futuro próximo se dotará a estos mareógrafos de la infraestructura necesaria para la recepción centralizada de datos.

La continua actividad de la flota hidrográfica requiere de la determinación del Cero Hidrográfico en los lugares donde se realiza un levantamiento hidrográfico, así como contar con datos de marea referidos a dicho nivel. Con este fin, utilizan las comisiones hidrográficas mareógrafos de presión portátiles. Uno de los principales avances tecnológicos que ha logrado el Instituto Hidrográfico radica en el sistema de calibración de estos mareógrafos. Dicho sistema, diseñado y construido en la Sección de Oceanografía, permite obtener una calibración tal que, en el rango de uso portuario, el error total, incluidos los errores de reloj, es inferior a los tres centímetros al 95\%. Este nivel de error es más pequeño que el requerido por la $\mathrm{OHI}$ para los levantamientos hidrográficos de zonas de especial peligro para la navegación.

El Comité de Mareas de la OHI, que cuenta con un miembro del Instituto Hidrográfico, redactó una nueva definición para el Cero Hidrográfico basada en las componentes armónicas. Dicha definición, apro- 
bada por la Conferencia Hidrográfica, plantea un problema en los puertos donde se realiza un nuevo levantamiento, puesto que requiere un año de observaciones de marea para alcanzar el nivel de precisión requerido. El Instituto Hidrográfico está desarrollando un método, basado en el cruce espectral con los puertos principales, que permite obtener el Cero Hidrográfico en los puertos secundarios, dentro de los niveles de precisión requeridos, a partir de cortos períodos de observación.

No menos importante que la anterior, también son misiones del Instituto Hidrográfico el apoyo a la Fuerza Naval y contribuir al progreso de la Oceanografía. La primera de ellas se realiza mediante la elaboración de informes ambientales, previos al comienzo de las operaciones y ejercicios, y el apoyo directo durante su desarrollo. En este año va a producirse una sustancial variación en esta actividad, puesto que, por primera vez, la Sección de Oceanografía va a actuar como Centro de Fusión de Datos en apoyo a un ejercicio internacional auspiciado por la Alianza Atlántica.

La búsqueda de la mejora de este apoyo ambiental y la contribución al progreso de la Oceanografía tienen tantos puntos en común, que proporcionan el marco idóneo para la colaboración con instituciones externas a la Armada, tanto nacionales como internacionales.

\section{Escuela de Hidrografía}

La Escuela de Hidrografía, ubicada dentro del IHM, tiene como misión fundamental la formación del personal hidrógrafo de la Armada en todas sus categorías, existiendo cuatro niveles según la función a desempeñar:.

- Oficial Ingeniero Hidrógrafo, para la dirección técnica de la actividad en las Secciones del Instituto Hidrográfico.

- Oficial Especialista en Hidrografía, para dirigir los levantamientos hidrográficos desde buques y comisiones hidrográficas.

- Suboficial Especialista Hidrógrafo, técnico a cargo de la ejecución de trabajos y operaciones con sistemas y equipos en buques e Instituto Hidrográfico.

- Personal de Marinería profesional de la especialidad de Maniobra y Navegación, como auxiliares en diferentes funciones en los levantamientos hidrográficos.

La formación de todo este personal se materializa a través de las siguientes categorías de cursos de Formación, Perfeccionamiento y Monográficos: 
- Cursos de Formación

Sólo existentes para el Acceso a la Escala de Suboficiales del Cuerpo de Especialistas, como especialidad fundamental.

De los dos años de formación para acceder al empleo de Sargento, el primero se desarrolla en común con el resto de las especialidades en la Escuela de Suboficiales; y el segundo se realiza íntegramente en la Escuela de Hidrografía.

Este curso está reconocido desde 1.997 por el Comité Consultivo de la Federación Internacional de Topógrafos y por la Organización Hidrográfica Internacional, dentro de la categoría «B» de Hidrógrafos.

A él asisten regularmente suboficiales de países hispanoamericanos y del norte de África, beneficiarios de becas del Ministerio de Defensa.

- Cursos de Perfeccionamiento

- Ingeniero Hidrógrafo, con una duración de dos años, para oficiales del Cuerpo General, Escala Superior, que sean ya especialistas en Hidrografía.

La enseñanza está orientada a la especialización en alguna de las ramas de la actividad del Instituto. Generalmente consiste en un primer año en el Real Observatorio de la Armada, donde se les imparten materias de ciencias y metodología, así como de medios y procedimientos para la investigación, y un segundo año concertado con las Universidades de Jaén y Cádiz donde cursan estudios de $2^{\circ}$ y/o $3^{\circ}$ ciclo de las carreras de «Ingeniero Superior en Geodesia, Cartografía y Fotogrametría» y «Ciencias del Mar», respectivamente.

También se cursan «Master» en diferentes Universidades extranjeras, según las necesidades del IHM.

- Oficiales Especialistas en Hidrografía, con una duración de diez meses, para oficiales del Cuerpo General y del Cuerpo de Especialistas, dividido en dos fases: un bloque teórico de la especialidad en la Escuela de nueve meses, y un mes de prácticas de fin de curso, donde se elabora el levantamiento completo de un parcelario.

Este curso está acreditado desde 1.987 como de Hidrógrafo categoría «A», por el Comité Consultivo FIG/OHI mencionado an- 
teriormente, lo que supone un reconocimiento al máximo nivel internacional.

- Aptitud de Hidrografía y Cartografía para Cabos $1^{\circ}$ y Cabos. Se imparte en la actualidad con una duración de 80 días lectivos, para Cabos procedentes de la Especialidad Fundamental de Maniobra y Navegación.

- Aptitud de Hidrografía Elemental para Marineros. Se imparte en la actualidad con una duración de 60 días lectivos, para marineros profesionales procedentes de la Especialidad Fundamental de Maniobra y Navegación.

- Otros cursos.

En esta Escuela se imparten también diversos cursos monográficos relacionados generalmente con la detección submarina y el posicionamiento de precisión, aspectos de gran importancia para determinadas funciones específicas:

- Sistemas de Posicionamiento

- Sistema Hidrográfico Integrado de la Marina Española (SHIME), para personal hidrógrafo..

- Sonar de Barrido Lateral.

- Acústica, Propagación y Predicción de Alcances Sonar.

Estos cursos tienen una duración que oscila entre treinta y cuarenta horas y están orientados a oficiales y suboficiales de Hidrografía, Fuerza MCM y Flota.

\section{A modo de epílogo}

Para finalizar esta presentación del IHM parece conveniente resaltar algunos aspectos de sus múltiples actividades, tanto a nivel nacional como internacional.

En primer lugar, que por Ley 7/1986 de Ordenación Cartográfica, se determina que será competencia del IHM la formación y conservación de la cartografía básica, con lo que se confiere a su producción la categoría de Cartografía de Estado y de documentación oficial de preceptiva utilización para el navegante.

Destacar de nuevo que el IHM representa a España en la Organización Hidrográfica Internacional, con sede en Mónaco, y participa activamente en la Comisión Hidrográfica del Mediterráneo y Mar Negro, en la Comisión Cartográfica de la Región Antártica, y en varios Comités 


\section{El Instituto Hidrográfico de la Marina}

(Carta Electrónica, Radioavisos, Sistemas de Información, etc..) y Grupos de Trabajo (Naváreas, Diccionario Hidrográfico, Planificación, etc...)

Asimismo, forma parte del Comité de Seguridad Marítima y del Subcomité de Seguridad de la Navegación y Radiocomunicaciones SAR, ambos pertenecientes a la Organización Marítima Internacional (OMI).

Dentro de la OTAN, el IHM participa en varios grupos de trabajo, destacando el MILOC y el Comité Científico de SACLANTCEN.

En cuanto a organizaciones nacionales, forma parte de varias comisiones y grupos, entre los que cabe destacar:

- Consejo Superior Geográfico

- Comisión española de Geodesia y Geofísica

- Comisión nacional de Faros y Señales Marítimas.

Por último, parece oportuno señalar que para llevar a cabo toda esta labor, el IHM cuenta con el siguiente personal civil y militar:

- 30 oficiales de todos los cuerpos y escalas

- 59 suboficiales de varias especialidades.

- 36 cabos y marineros.

- 40 funcionarios civiles

- 129 civiles (personal laboral)

\section{Notas}

* ED 50: European Datum (año 1950). WGS 84: World Geodesic System (año 1984) 